\title{
Major upgrades of the high frequency B-dot probe diagnostic suite on ASDEX Upgrade
}

\author{
Roman Ochoukov ${ }^{1, *}$, Volodymyr Bobkov ${ }^{1}$, Cecilia Bruhn ${ }^{1,2}$, Rodolphe D'Inca ${ }^{1}$, Helmut Faugel $^{1}$, Helmut Fuenfgelder ${ }^{1}$, \\ Rachael McDermott ${ }^{1}$, Philip Schneider ${ }^{1}$, Guillermo Suarez-Lopez ${ }^{1,3}$, Jean-Marie Noterdaeme ${ }^{1,4}$, \\ ASDEX Upgrade Team ${ }^{* *}$ and MST1 Team*** \\ ${ }^{1}$ Max Planck Institute for Plasma Physics - Garching, Germany \\ ${ }^{2}$ Technical University of Munich - Garching, Germany \\ ${ }^{3}$ Ludwig Maximilian University - Munich, Germany \\ ${ }^{4}$ Ghent University - Ghent, Belgium
}

\begin{abstract}
The high frequency B-dot (HFB) probe diagnostic on the ASDEX Upgrade tokamak has undergone a considerable upgrade during the 2016 opening of the torus. The probe coverage is now greatly expanded toroidally, as well as radially with the addition of probes on the high field side and the removable manipulator head. A new 2-channel fast digitizer now allows to examine and record radio frequency (RF) wave emissions emanating from the plasma in the ion cyclotron range of frequencies (ICRF). Possible studies that can be achieved now include: a study of core ICRF power absorption efficiency; a study of ion cyclotron emissions from the plasma generated by energetic ions; and study of ICRF wave/plasma turbulence interactions in the scrape-off layer region.
\end{abstract}

\section{Introduction}

Radio frequency waves in the ion cyclotron range of frequencies (ICRF) are successfully used to heat magnetized plasmas to fusion relevant temperatures. Examples of tokamaks using ICRF systems are ASDEX Upgrade [1], Alcator C-Mod [2], Tore Supra/WEST [3], and JET [4]. ITER, the next generation tokamak [5], and DEMO, the first generation fusion power plant [6], are also going to rely on ICRF power for their operation. The ICRF system on ASDEX Upgrade is tasked with studying ICRF power coupling and mitigating unwanted plasmamaterial interactions, while maintaining high performance core plasma [1,7]. In order to assist with this task, a number of probes have been installed that directly detect the ICRF waves' magnetic field components and their properties. This set of high frequency B-dot (HFB) probes contains an array of inductors capable of measuring the amplitude, the phase, and the polarization of the ICRF wave [8]. It recently received an upgrade with a number of individual probes that greatly expanded the diagnostic coverage toroidally and radially with a number of probes placed on the high field side of the ASDEX Upgrade tokamak. Additionally, a high frequency 2channel digitizer has been added to the diagnostic rack, in order to resolve the ion cyclotron emission (ICE) frequency spectra emanating from the plasma. Finally, a pair of HFB probes, together with a pair of Langmuir probes, are put together for a new diagnostic head of the ASDEX Upgrade low field side midplane manipulator.
This additional diagnostic aims to measure the radial profile of the ICRF wave field amplitude in the scrape off layer (SOL), as well as to study the interactions between turbulent SOL plasmas and ICRF wave fields. The overall outline of the paper is to describe the details of the newly expanded HFB diagnostic and to demonstrate the capabilities of these new additions to measure ICRF wave properties in various plasma conditions. Possible future studies will also be suggested.

\section{Diagnostic and Experimental Procedure Description}

The first set of HFB probes was installed before the 2015 ASDEX Upgrade experimental campaign and included three probe pairs and one field-aligned probe array of six probe pairs, all located on the low field side near the midplane [8]. Each pair consists of two inductors, orthogonally aligned to each other: one probe oriented to measure the fast wave polarization and the other for the slow wave. An example of a single probe, with the inductor exposed is shown in Fig. 1 (a). The latest addition for the 2017 campaign now includes 8 new probe pairs: 4 on the low field and 4 on the high field side. Another probe pair is installed, together with a pair of Langmuir probes, on a removable diagnostic head for the ASDEX Upgrade midplane manipulator (MEM), Fig. 1 (b). The purpose of the additional probes on the high field side is to help quantify the efficiency of various ICRF absorption schemes: hydrogen (H-) or helium-3 $\left({ }^{3} \mathrm{He}-\right)$ minority

\footnotetext{
*Corresponding author: rochouko@ipp.mpg.de

${ }^{* *}$ For authors list, see H. Zohm et al., Nucl. Fusion 55, 104010 (2015).

${ }^{* * *}$ For authors list, see H. Meyer et al., Nucl. Fusion Special issue, Nucl. Fusion 57 (2017) 102014.
} 

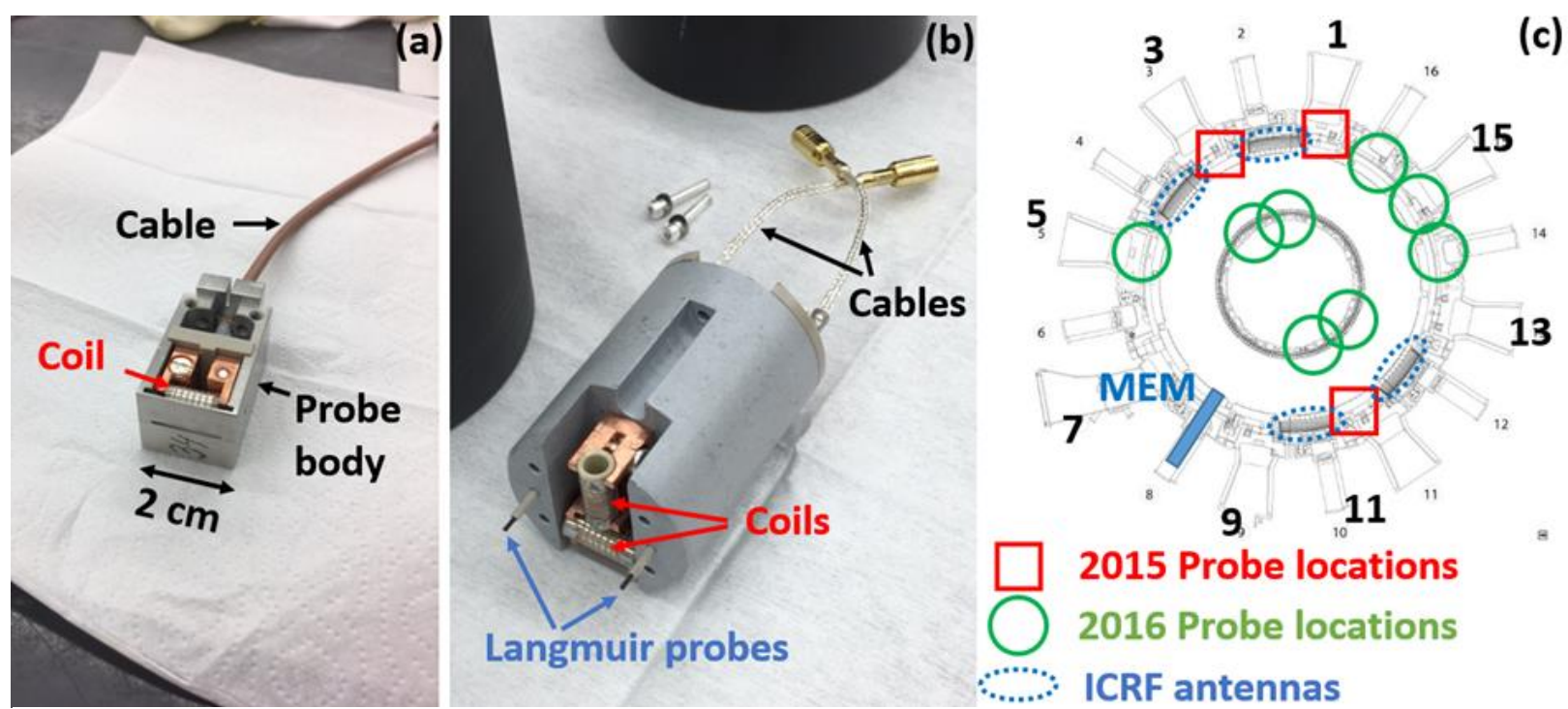

(c)

Fig. 1. (a) the key components of a single HFB probe; (b) the key components of the midplane manipulator (MEM) ICRF head; (c) the locations of the previous and latest installations of the HFB probes. The ICRF antennas and the MEM toroidal positions are also indicated.

heating, 3-ion heating, or higher harmonic D heating. The new MEM probe head will be used to study interactions between ICRF waves and SOL turbulence. The ICRF wave amplitude signal is generally sampled with a logarithmic RF detector that cannot distinguish frequencies in the range of $10-50 \mathrm{MHz}$ [8]. However, the addition of a 2-channel ICE detector/digitizer with a sampling frequency of $125 \mathrm{MHz}$ allows us to measure emitted ICE frequency spectra up to $67.5 \mathrm{MHz}$ from two different probes simultaneously. The overall layout of the probes inside the ASDEX Upgrade torus is shown in Figure 1 (c) .

Both L- and H-mode discharges are used for ICRF studies with the HFB probes. The H-mode is typically achieved on ASDEX Upgrade when the total auxiliary heating power is in excess of $2 \mathrm{MW}$, which can be reached with a combination of ICRF, electron cyclotron waves, and neutral beam injection. The ICRF power is typically injected at either $30 \mathrm{MHz}$ (for the on-axis toroidal field value $\mathrm{B}_{\mathrm{T}}=+/-2 \mathrm{~T}$ ) or $36.5 \mathrm{MHz}$ (for $\mathrm{B}_{\mathrm{T}}=+/-2.5 \mathrm{~T}$ ), with hydrogen minority being the dominant heating scheme in these discharges. Fig. 1 (c) shows the locations of the ICRF antennas inside the ASDEX Upgrade torus.

To determine how efficiently ICRF power is absorbed in the plasma core, we use the break-in-slope method [9], while modulating ICRF power on/off with the frequency of 8-10 Hz. Following this method the ICRF power absorption coefficient ( $\eta_{\mathrm{ICRF}}$ ) is estimated from the rate of change of the stored plasma energy $\left(\mathrm{dW}_{\mathrm{MHD}} / \mathrm{dt}\right)$ before and after the ICRF pulse is turned off: $\eta_{\text {ICRF }} \cong$

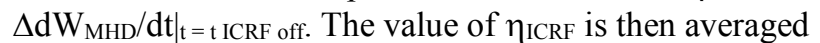
over 5-10 ICRF pulses to smoothen the effect of edge localized modes (ELMs) on the estimate. The goal is then to determine if the expected trend (weaker RF field amplitude $\left(\mathrm{V}_{\mathrm{RF}}\right)$ on the high field side for stronger ICRF absorption coefficient) is observed in ICRF-heated discharges on ASDEX Upgrade. It is worth noting that the break-in-slope method underestimates $\eta_{\text {ICRF. }}$ The method assumes a prompt plasma response, which is not entirely true for the H-minority heating scheme, where a large power fraction is absorbed on the ions and only then is transferred to the electrons through collisions [9].

\section{Experimental Results and Discussion}

The break-in-slope technique is commonly used to determine how efficiently ICRF power is absorbed in the plasma core [9]. In our case, the ICRF power was modulated at either 8.333 or $10.0 \mathrm{~Hz}$ (Fig. 2 (a)). The corresponding changes in the plasma stored energy are approximated with linear curves (Fig. 2 (b)), the slopes of which are then used to estimate $\eta_{\text {ICRF. }}$ The core plasma density remains constant during these modulations (Fig. 2 (c)), excluding a possible effect on plasma fueling by the active antennas. The modulation of the core soft x-ray (SXR) emissions and the neutron rate $(\mathrm{dN} / \mathrm{dt})$ are in line with ICRF power being deposited in the plasma core and heating the plasma species (Figs. 2 (d) and (e)). The SXR signal is additionally modulated by sawteeth at nearly the same frequency as the ICRF power modulation (Fig. 2 (d)). The strength of the ICRF fast wave amplitude on the high field side is then recorded during these modulations (Fig. 2 (f)) to compare with the ICRF power absorption coefficient.To smoothen the effect of ELMs, which are clearly visible on the time traces of $\mathrm{W}_{\mathrm{MHD}}$ (Fig. 2 (b)) and $\mathrm{V}_{\mathrm{RF}}$ (Fig. 2 (f)), we use 5-10 ICRF pulses to obtain a single average $\eta_{\text {ICRF }}$ value. The expected trend is to have a lower ICRF fast wave amplitude on the high field side, as the ICRF power absorption coefficient improves. Such behavior is observed (Fig. 3). In total, six discharges (five $\mathrm{H}$ - and one L-mode) were used, where each point represents a single on/off ICRF cycle. Note that the Hminority concentration is $\sim 4-6 \%$ in these discharges, suggesting that the reason for the higher $\mathrm{V}_{\mathrm{RF}}$ and lower $\eta_{\text {ICRF }}$ in $\mathrm{H}$-mode discharges with ICRF power at 30.0 $\mathrm{MHz}$ is due to a different wavenumber spectrum of the launched ICRF wave.

The ability to study ICRF power absorption is not the only capability of the newly expanded HFB diagnostic suite. With the addition of a fast 2-channel digitizer 


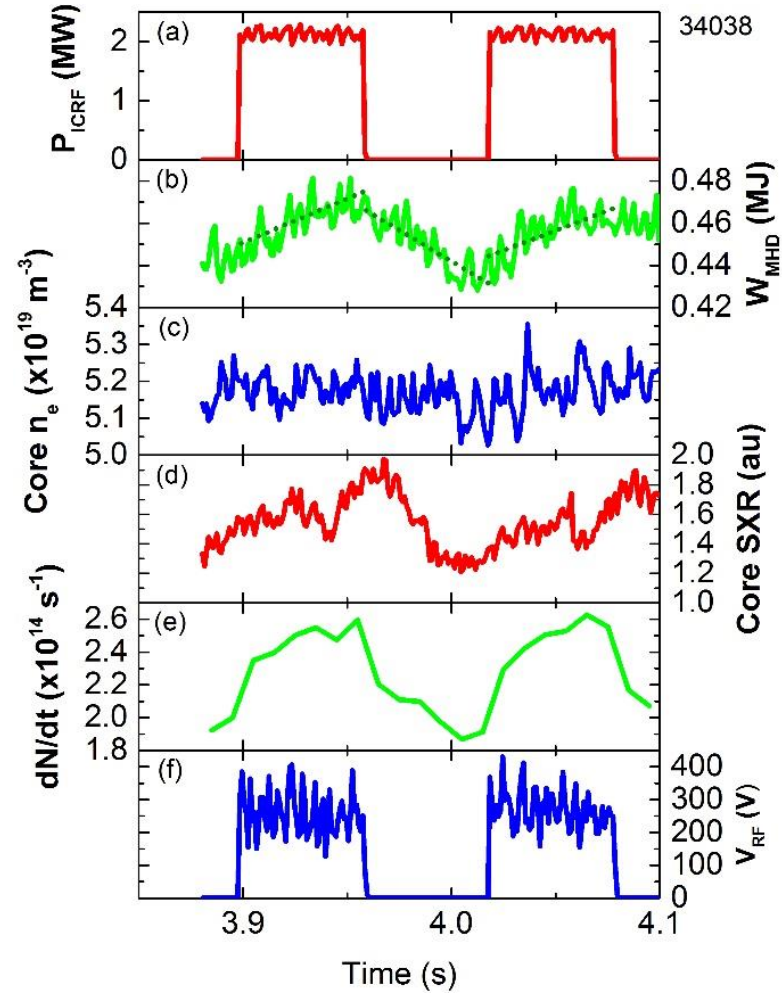

Fig. 2. The key plasma and machine parameters of a discharge used to examine core ICRF wave absorption. (a) the ICRF power PICRF; (b) the stored plasma energy $\mathrm{W}_{\mathrm{MHD}}$; (c) the core plasma density ne; (d) the soft $\mathrm{x}$ ray (SXR) signal from a chord that passes through the plasma core; (e) the neutron rate $\mathrm{dN} / \mathrm{dt}$; and (f) the fast ICRF wave amplitude $V_{R F}$ from the high field side HFB probe in Sector 2. The dashed lines in (b) are linear fits.

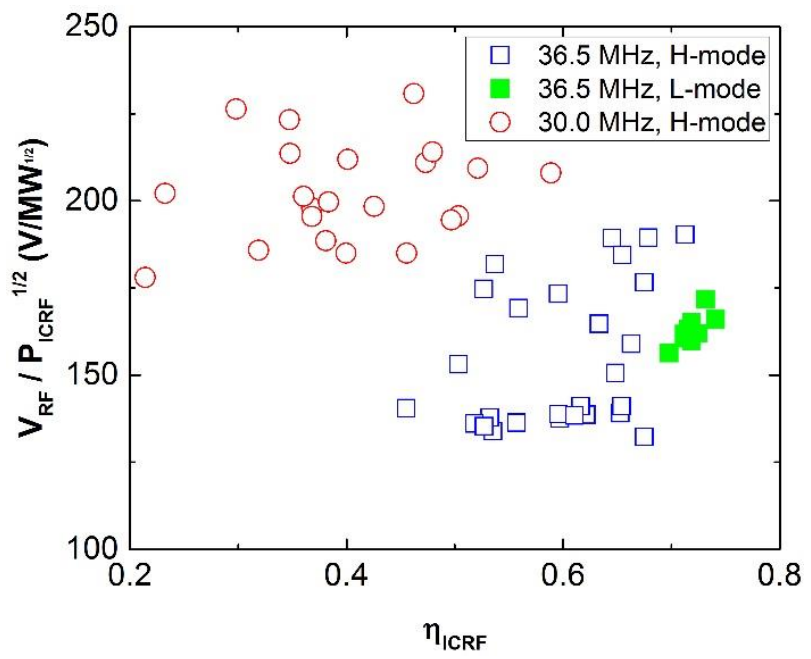

Fig. 3. The normalized fast wave amplitude $V_{R F}$ from the Sector 2 high field side probe, as a function of the estimated ICRF power absorption coefficient $\eta_{\text {ICRF. }} \eta_{\text {ICRF }}$ is estimated using the break-in-slope method [9]. Note the the break-in-slope method underestimates $\eta_{\mathrm{ICRF}}$ for the case of H-minority heating [9].

system, it is now possible to measure ICE frequency spectra emanating from the magnetized tokamak plasma in the presence of energetic ions. An example of one ICE type is obtained from one of the shots that are used for the ICRF power absorption study (Fig. 4). The $10 \mathrm{~Hz}$ power "blips" of ICRF operated at $36.5 \mathrm{MHz}$ are clearly visible, as well as the aliased second harmonic at $52.0 \mathrm{MHz}$. We,

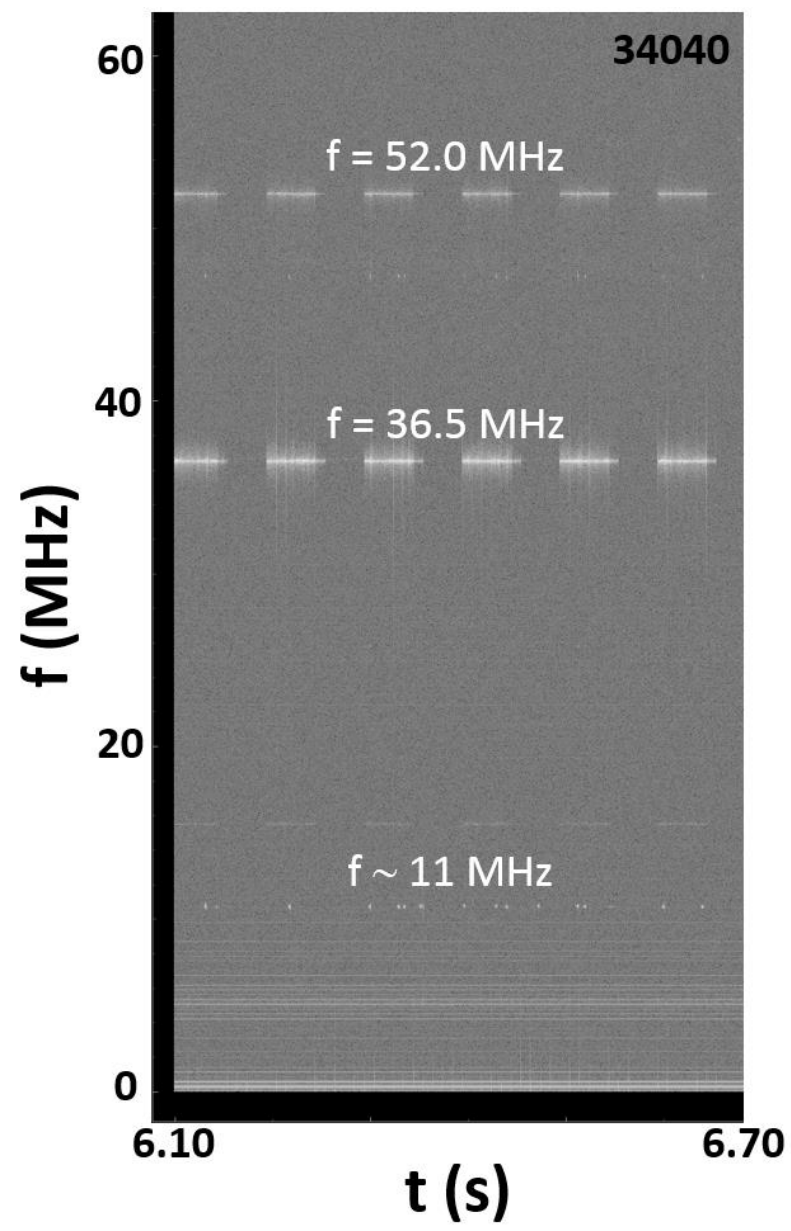

Fig. 4. An example of the ion cyclotron emission (ICE) spectrum on ASDEX Upgrade. The ICE signal at $\sim 11 \mathrm{MHz}$ is attributed to the energetic deuterium ions, produced by neutral beam injections at $60 \mathrm{kV}$ grid bias.

additionally, observe short bursts at $\sim 11 \mathrm{MHz}$ and these are generally attributed to the neutral beam deuterium ions exciting magnetoacoustic cyclotron wave emissions from the low field side [10]. The on/off bursts of the emission are correlated with the ELMs. We also see uninterrupted "emission" lines below $10 \mathrm{MHz}$, which are the internal electronics noise of the diagnostic.

$\mathrm{H}$ - and L-mode discharges shown so far are not the only tokamak plasma modes that are studied for the purpose to provide favourable fusion environment. The so-called I-modes, which have beneficial transport properties [11], are also of interest on ASDEX Upgrade. One of the defining features of the I-mode is the appearance of a mode in the plasma pedestal region, with a characteristic frequency of $\sim 100 \mathrm{kHz}$. Such a feature is, indeed, observed with a newly installed MEM diagnostic head, equipped with a pair of HFB probes (as well as a pair of Langmuir probes) (Fig. 5). The manipulator was plunged for the duration of 2.8-3.2 s, to coincide with the appearance of an I-mode (Fig. 5 (a)). The high frequency mode is visible as a modulation of the ICRF wave amplitude with a broad local maximum at $\sim 100 \mathrm{kHz}$ (Fig. 5 (b)). The feature is commonly called the weakly coherent mode, or WCM [11]. Note, that the I-mode was "sampled" by the manipulator probe in the inserted position at the major radius of the limiter, while the L- and 


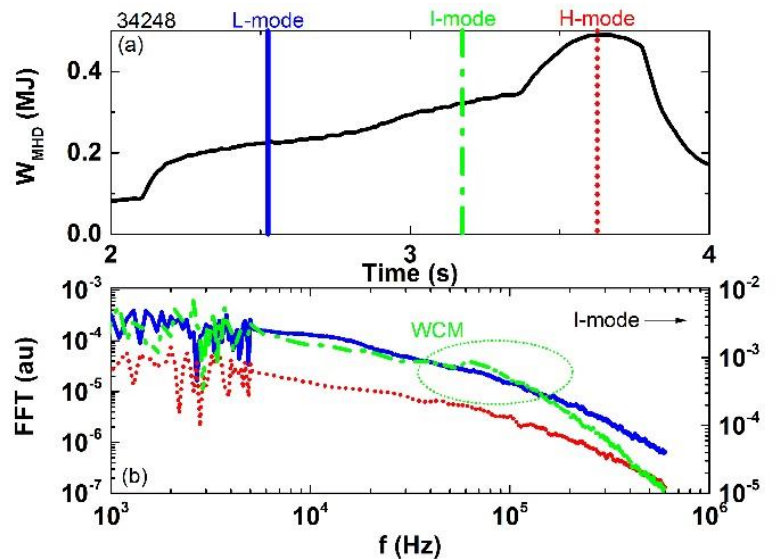

Fig. 5. (a) The stored plasma enegry $\mathrm{W}_{\text {MHD }}$ is used to identify three different plasma modes, the L-, the I-, and the H-modes. (b) The frequency spectra of the ICRF fast wave amplitude measured with the MEM diagnostic head in the three plasma modes. The WCM refers to the broad weakly coherent mode observed near $100 \mathrm{kHz}$ in the I-mode part of the discharge.

$\mathrm{H}$-mode data were obtained in the retracted position of the manipulator (10 $\mathrm{cm}$ behind the limiter).

\section{Conclusion}

The HFB diagnostic on ASDEX Upgrade has been greatly expanded during the 2016 opening. The expansions include additional probes that increase the toroidal and radial diagnostic coverage, as well as the additional ability to measure frequency spectra emitted by the plasma in the ICRF range. Possible studies with the new HFB diagnostic suite include the efficiency of core ICRF wave absorption in different heating schemes (Fig. 3) or identification of ion cyclotron emissions under various plasma conditions (Fig. 4). It is also capable of providing information on ICRF wave-plasma turbulence interactions in the SOL and pedestal plasma regions with the new midplane manipulator head (Fig. 5).

This work has been carried out within the framework of the EUROfusion Consortium and has received funding from the Euratom research and training programme 2014-2018 under grant agreement No 633053. The views and opinions expressed herein do not necessarily reflect those of the European Commission.

\section{References}

1. V. Bobkov, F. Braun, R. Dux, et al., Nucl. Fusion Nucl. Fusion 56 (2016) 084001.

2. S. J. Wukitch, M. L. Garrett, R. Ochoukov, et al., Physics of Plasmas 20, 056117 (2013).

3. B. Soutic, et al., Plasma Phys. Control. Fusion 36 (1994) B123-B132.

4. F. Durodié, M. Nightingale, A. Argouarch, et al., Fusion Engineering and Design 84 (2009) 279283.

5. D. Milanesio and R. Maggiora, Nucl. Fusion 50 (2010) 025007.
6. A. Bader, G. Bosia, A. Messiaen, et. al., Fusion Engineering and Design, in press (2017).

7. W. Zhang, V. Bobkov, T. Lunt, et. al., Nucl. Fusion 56 (2016) 036007.

8. R. Ochoukov, V. Bobkov, H. Faugel, H. Fünfgelder, et. al., Review of Scientific Instruments 86, 115112 (2015).

9. E. A. Lerche, D. Van Eester and JET EFDA Contributors, Plasma Phys. Control. Fusion 50 (2008) 035003.

10. R. O. Dendy, K. G. McClements, C. N. Lashmore-Davies, et al., Phys. Plasmas 1 (10), 1994.

11. D. G. Whyte, A. E. Hubbard, J. W. Hughes, et al., Nucl. Fusion 50 (2010) 105005. 